\begin{tabular}{|c|c|}
\hline Title & A coustic phonon modes of rectangular quantum wires \\
\hline Author(s) & Nishiguchi, N.; A ndo, Y.; Wybourne, M. N. \\
\hline Citation & $\begin{array}{l}\text { Journal of Physics: Condensed Matter, } 9(27), 5751-5764 \\
\text { https://doi.org/10.1088/0953-8984/27/007 }\end{array}$ \\
\hline Issue Date & 1997 \\
\hline Doc URL & http:/hdl.handle.net/2115/49626 \\
\hline Rights & Copyright @ 1997 IOP Publishing Ltd. \\
\hline Type & article (author version) \\
\hline File Information & J Phys Condens Matter_9_27_1997.pdf \\
\hline
\end{tabular}

Instructions for use 


\title{
Acoustic phonon modes of rectangular quantum wires
}

\author{
N. Nishiguchi $\dagger$, Y. Ando $\dagger$ and M. N. Wybourne $\ddagger$ \\ $\dagger$ Department of Applied Physics, Hokkaido University, Sapporo 060, Japan \\ $\ddagger$ Department of Physics, University of Oregon, Eugene, Oregon 97403, U.S.A.
}

\begin{abstract}
Acoustic phonon modes of a free-standing rectangular quantum wire of cubic crystals are theoretically investigated using an algorithm developed to analyze data from resonant ultrasound spectroscopy. The normal phonon modes are classified by their spatial symmetries into a compressional mode termed the dilatational mode and non-compressional modes referred to as the flexural, torsional and shear modes. The formalism we present is quite general and can be applied to wires of any cubic material. As an example, the dispersion relations are obtained for square and rectangular wires of GaAs, taking into account anisotropic elasticity of the material. The dispersion curves for a rectangular wire are compared with those of the approximate hybrid modes referred to as the thickness and width modes, and the validity of the modes is discussed. The existence of edge modes is confirmed by examining the spatial distribution of displacement vectors.
\end{abstract}

PACS numbers: PACS Numbers:63.20.Dj,72.10.Di,73.20.Dx

\section{Introduction}

A number of theoretical and experimental studies have investigated transport phenomena in nanometer scale wires at temperatures where the acoustic phonons are expected to show reduced dimensional behavior. Such behavior is anticipated in wires whose cross-sectional dimension is less than the phonon phase coherence length. On this length scale, phonon confinement will result in nonlinear dispersion and acoustic subbands at wavevectors close to the Brillouin zone center.[1] The effects of confinement on the phonon mean-free-path,[2] thermal conductivity, [3, 4] and phonon localization[5] have received some attention. Other work has addressed electron transport in the presence of phonon confinement. In experiments on semiconductor wires no confinement related effects have been found.[6] In other experiments, the resistance of metal wires was observed to change as electron energy relaxation to confined modes became possible.[7, 8] An issue related to these studies is the nature of the phonon spectrum near the zone center. Of particular importance to wires of rectangular cross-section is an understanding of edge modes which are expected to become increasingly important as the cross-sectional dimensions of a wire are reduced. 
Theoretical work on phonons in quasi one-dimensional(Q1D) quantum wires has advanced relatively slowly in contrast to the case of electrons in the quantum wires or that of phonons in higher dimensional systems such as superlattice structures. One of the main reasons is that even for rectangular wires the longitudinal(LA) and transverse acoustic (TA) wave coupling that occurs at the wire surface makes it difficult to derive the normal phonon modes and their spectra analytically. Previous theoretical approaches have been limited to specific geometries, such as circular or very flat rectangular wires of elastically isotropic materials, and have provided only a qualitative description of the phonon dispersion in realizable quantum wires. Since the combination of the LA and TA waves depends on geometry, frequency and wavevector, [9] it is difficult to determine the LA wave component using approximate methods. This issue has made estimates of the deformation potential scattering of electrons in wires uncertain, and has shown that to model the low energy electron scattering rates in nanostructures accurately, a more complete treatment of the confined acoustic modes is required. $[10,11]$

Recently, an accurate method was developed in the resonant ultrasound spectroscopy $[12,13,14]$ to derive free vibrational modes of inhomogeneous objects. This method can treat free vibrations of a general anisotropic object with arbitrary shape as well as arbitrary mass density variation, by expanding the displacement vectors with a set of basis functions of the products of powers of the Cartesian coordinates, which is called the $x y z$ algorithm.[13] Considering the translational symmetry of wires along the wire axis, vibrations of free-standing wires reduce to free vibrations of flakes, which can be analyzed by the method. Thus the $x y z$ algorithm is found to be useful for the normal modes of free-standing wires of anisotropic materials with arbitrary shape cross section, by suitably choosing the basis functions of displacement vectors.

In this paper, we show that the acoustic phonon modes of Q1D wires can be precisely obtained by the $x y z$ algorithm. The plan of this paper is as follows: In Sec. II, we outline the method and apply it to a rectangular wire of a cubic material. The normal phonon modes are classified into the dilatational, flexural, torsional, and shear modes, according to the symmetries of the modes. In Sec. III, we apply the results of Sec. II to wires of the cubic semiconductor GaAs. We have chosen this material as an example because the normal modes can be compared with previously published calculations.[10] We note, however, that our approach is quite general and can be applied to wires of any cubic material. We determine the dispersion curves and show their dependence on the crosssectional aspect ratio. We also compare the results with previous approximate hybrid modes termed the thickness and width modes $[15,16]$ calculated assuming separable boundary conditions. In Sec. IV, we examine the spatial distribution of displacements and show the existence of edge modes having large amplitudes at the wire corners. The normalization condition is applied to the acoustic phonon modes of rectangular wires, and the phonon field is formulated in Sec. V. A summary is given in Sec. VI. 


\section{Method}

In this section, we derive an eigenvalue equation for acoustic phonon modes of a freestanding wire based on the variational principle. The Lagrangian of the system is given in terms of the displacement components $u_{i}$ and angular frequency $\omega$, by

$$
L=\int_{V}\left[\frac{\rho}{2} \omega^{2} u_{i} u_{i}-\frac{1}{2} C_{i j k \ell} \partial_{i} u_{j} \partial_{k} u_{\ell}\right] d V,
$$

where $\rho$ and $C_{i j k \ell}$ are the mass density and stiffness tensor of the constituent material. $V$ denotes the volume of the wire. The variation of the Lagrangian $\delta L$ due to the change of the displacement components $u_{i} \rightarrow u_{i}+\delta u_{i}$ yields

$$
\delta L=\int_{V}\left[\rho \omega^{2} u_{i}+\partial_{j} \sigma_{j i}\right] \delta u_{i} d V-\int_{S} n_{j} \sigma_{j i} \delta u_{i} d S,
$$

where $n_{j}$ is the $j$ th component of the unit vector normal to the wire surface $S$ and $\sigma_{i j}$ is the stress tensor given by

$$
\sigma_{i j}=C_{i j k \ell} \partial_{k} u_{\ell}
$$

For a free-standing wire, the stress field vanishes at the wire surface, i.e.

$$
\left.\sigma_{i j} n_{j}\right|_{S}=0 \text {. }
$$

Considering the boundary condition (4) and postulating that the Lagrangian has the minimum with respect to $u_{i}$, we obtain the following elastic wave equation,

$$
\rho \omega^{2} u_{i}+\partial_{\ell} \sigma_{\ell i}=0 \text {. }
$$

Expanding the displacement components $u_{i}$ by means of a complete set of functions $\left\{\Phi_{\lambda}\right\}$

$$
u_{i}=\chi_{i \lambda} \Phi_{\lambda}
$$

and substituting Eq. (6) into (5), we obtain

$$
\chi_{i \lambda} \rho \omega^{2} \Phi_{\lambda}+\chi_{\ell \lambda} C_{i j k \ell} \partial_{j} \partial_{k} \Phi_{\lambda}=0 .
$$

Multiplying Eq. (7) by $\Phi_{\lambda^{\prime}}^{*}$ and integrating over the volume $V$, we get the following generalized eigenvalue equation

$$
\chi_{i \lambda} \rho \omega^{2} \int_{V} \Phi_{\lambda^{\prime}}^{*} \Phi_{\lambda} d V-\chi_{\ell \lambda} C_{i j k \ell} \int_{V} \partial_{j} \Phi_{\lambda^{\prime}}^{*} \partial_{k} \Phi_{\lambda} d V=0 .
$$

Defining the matrix elements $E_{i \lambda^{\prime} ; j \lambda}$ and $\Gamma_{i \lambda^{\prime} ; \ell \lambda}$ by

$$
E_{i \lambda^{\prime} ; j \lambda}=\delta_{i, j} \frac{\rho}{V} \int_{V} \Phi_{\lambda^{\prime}}^{*} \Phi_{\lambda} d V
$$

and

$$
\Gamma_{i \lambda^{\prime} ; \ell \lambda}=\frac{C_{i j k \ell}}{V} \int_{V} \partial_{j} \Phi_{\lambda^{\prime}}^{*} \partial_{k} \Phi_{\lambda} d V
$$

Eq. (8) can be expressed in a matrix form as

$$
\left[\omega^{2} \mathbf{E}-\boldsymbol{\Gamma}\right] \chi=0 .
$$


Normal phonon modes are obtained by solving Eq. (11). Eigenvalues $\omega_{J}^{2}$ of Eq. (11) to give the normal mode spectra, and the corresponding displacement vector $\mathbf{u}_{J}$ is given by Eq. (6) where the coefficients $\chi_{i \lambda}$ are substituted by the eigenvector components $\chi_{J, i \lambda}$. Here the subscript $J$ denotes a set of quantum numbers to specify the normal modes.

The eigenvalue equation (11) is applied to a Q1D wire of elastically anisotropic materials with arbitrary shape of cross section. In what follows, we study acoustic phonon modes in a rectangular wire of a cubic crystal, by specifying a set of basis functions. Taking the wire axis to be the $z$ direction, the simplest basis functions are powers of the Cartesian coordinates[13] in the lateral directions such as

$$
\Phi_{\lambda}(x, y, z)=\left(\frac{2 x}{W}\right)^{m}\left(\frac{2 y}{H}\right)^{n} e^{i q z},
$$

where $\lambda=(m, n)$ and $q$ is the longitudinal wavevector of acoustic phonon modes along the wire axis. $W$ and $H$ are the thickness of the wire in the $x$ and $y$ directions, respectively, with the origin being the wire axis at the center of the cross section.

Substituting Eq. (12) into (9) and (10), yield the matrix elements,

$$
E_{i \lambda^{\prime} ; j \lambda}=\delta_{i, j} \rho F\left(m+m^{\prime} ; n+n^{\prime}\right)
$$

where

$$
F(m ; n)=\delta_{m, \text { even }} \delta_{n, \text { even }} \frac{1}{(m+1)(n+1)},
$$

and $\Gamma_{i \lambda^{\prime} ; \ell \lambda}$ given in Appendix A.

We note here that another complete set of functions such as products of the Legendre polynomials is also useful for the basis functions. The matrix $\mathbf{E}$ becomes diagonal in this case since the Legendre polynomials are orthogonal, and Eq. (11) results in the usual eigenvalue equation. Although the basis functions (12) are not orthogonal, they are linearly independent and are related to the Legendre polynomials by a certain linear transformation. Hence expanding the displacement with the basis functions (12) is essentially equivalent to that using the Legendre polynomials. One advantage of using the basis functions (12) is the straightforward evaluation of the $\boldsymbol{\Gamma}$ and $\mathbf{E}$ matrix elements, irrespective of the wire's cross sectional shape. Then this method can deal with wires with a variety of cross sectional shape. Another advantage is that the basis functions (12) are useful for classification of phonon modes as discussed below.

Table 1. A set of parity of displacement components

\begin{tabular}{cccc}
\hline Phonon Mode & $u_{x}$ & $u_{y}$ & $u_{z}$ \\
\hline I & $(-,+)$ & $(+,-)$ & $(+,+)$ \\
II & $(-,-)$ & $(+,+)$ & $(+,-)$ \\
III & $(+,+)$ & $(-,-)$ & $(-,+)$ \\
IV & $(+,-)$ & $(-,+)$ & $(-,-)$ \\
\hline
\end{tabular}


The basis functions have even or odd parity for inversion of $x$ and/or $y$ coordinates. Expressing the parity of the basis function (12) by $(\mu, \nu)$, where $\mu=(-1)^{m}$ and $\nu=(-1)^{n}$, the basis functions are classified into four parity groups, i.e. $(+,+)$, $(+,-),(-,+)$, and $(-,-)$. The diagonal matrix elements $\Gamma_{i \lambda^{\prime} ; i \lambda}$ connect among the basis functions belonging to the same parity group. In contrast, the off-diagonal matrix elements of $\Gamma_{i \lambda^{\prime} ; j \lambda}$ connect among the basis functions with different parity. Thereby, each displacement component $u_{i}$ consists of a set of basis functions belonging to one of the parity groups, and the displacement components $u_{x}$ and $u_{y}$ connected to the $u_{z}$ with the parity $(\mu, \nu)$ have parity $(-\mu, \nu)$ and $(\mu,-\nu)$, respectively. Normal phonon modes are described by the combination of these displacement components with different parity, and are classified into four kinds of vibrational modes with different spatial symmetries (Table I). The displacement components for each mode, which we numbered I-IV for convenience, are given in Appendix B.

We note that mode I is the dilatational mode, modes II and III are flexural modes, and mode IV is the torsional or shear modes as understood from Table I and Eqs. (Appendix B), (Appendix B), (Appendix B) and (Appendix B). Considering the change of cross sectional area for these modes, only the dilatational mode is a compressional mode.

Although a large number of basis functions are needed for the exact expression of normal modes, a small number of basis functions are enough for the expression as far as low frequency normal modes are concerned. The size of a truncated set of the basis functions is limited by the maximum power $N$ of the basis functions in Eqs. (Appendix B), (Appendix B), (Appendix B), and (Appendix B), which is determined by studying the convergence of the dispersion relationships for the frequency range and crosssectional dimensions of interest.

\section{Dispersion relations}

Figures 1, 2, and 3 illustrate the dispersion curves of the dilatational(I), flexural(II and III), and torsional and shear modes(IV) for a GaAs square wire with cross sectional dimensions $100 \AA \times 100 \AA$, respectively, showing the phonon subband structures due to quantization of wavevectors in the lateral direction. For this size wire, and a maximum frequency of $0.5 \mathrm{THz}, N$ was determined empirically to be 12 . The number of basis functions used for modes I, II and III is 70, and for mode IV is 63 . It should be noted here that for square wires the dispersion relations of the two flexural modes are degenerate. For a small $q$, the lowest dispersion curves of the dilatational and torsional modes are proportional to $q$, while the flexural modes show $q^{2}$ dependence. The parabolic dispersion relations of the flexural modes stem from the fact that these modes are bending modes similar to antisymmetric Lamb waves of a free plate,[1] which can be analytically derived[17] in the long wavelength limit as $\omega=q^{2} \sqrt{Y I_{i} / \rho W H}$. Here $Y$ is Young's modulus, and $I_{i}$ is the moment of inertia of the cross section $I_{x}=H^{3} W / 12$ about $x$ axis for mode II and $I_{y}=H W^{3} / 12$ about $y$ axis for mode III. The parabolic 


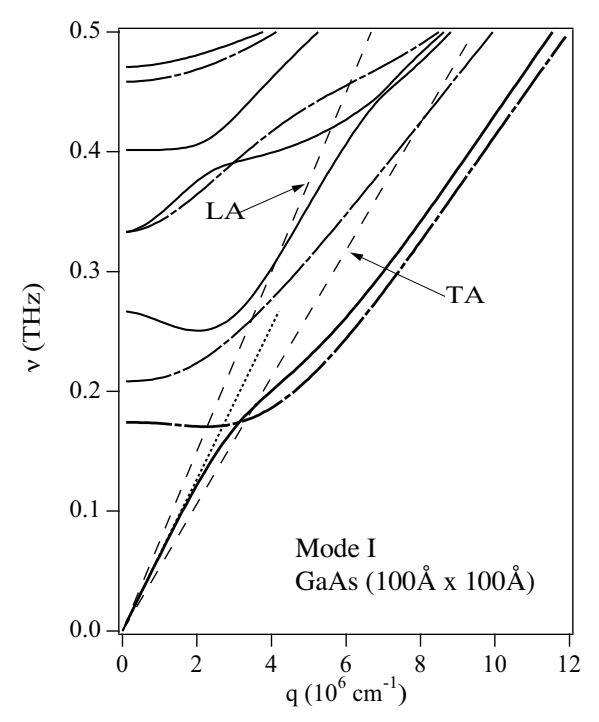

Figure 1. Dispersion curves of the dilatational phonon mode of a GaAs square wire with cross sectional dimensions $100 \AA \times 100 \AA$. The solid and dot-dashed lines denote the dispersion curves of the mode with vibrational patterns (a) and (b) in Fig. 4, respectively. The dashed lines are the dispersion curves of the bulk LA and TA waves along the [001] direction. The dotted line is the dispersion curve of a longitudinal wave in a rod in the long wavelength limit. The material parameters used are quoted from Ref. [21].

dispersion relation denoted by the dotted curve in Fig. 2 reproduces the lowest dispersion curves of the flexural modes below $q W=1$. On the other hand, the propagation velocity of the lowest subband of the dilatational mode is given by $\sqrt{Y / \rho}$, which is smaller than that of the bulk LA waves $\sqrt{C_{11} / \rho}$. The dispersion curve in the long wavelength limit is also plotted by the dotted line in Fig. 1. The propagation velocity of the torsional mode yields $v=\sqrt{G / \rho I_{z}}$, where $G$ and $I_{z}$ are the torsional rigidity and the moment of inertia of the cross section about the wire axis given by $I_{z}=I_{x}+I_{y}$, respectively. The dispersion curve is denoted by the dotted line in Fig. 3. Thus the lowest dispersion curves show the peculiarities of vibrational patterns of the normal modes.

For a larger $q$ or in a higher frequency region, some dispersion curves of the dilatational mode intersect, which does not occur for the other modes. The intersection comes about because the dilatational mode consists of two independent modes: one is a simple dilatation and contraction as schematically shown in Fig. 4(a), whose displacement vector $\mathbf{u}$ is transformed as $\mathbf{u}=C_{4} \mathbf{u}$ with rotational operation of the wire by $\pi / 2$ about the wire axis, and the other is the alternate dilatation and contraction of thickness of the wire in the $x$ and $y$ directions as shown in Fig. 4(b). The displacement vector is transformed as $\mathbf{u}=-C_{4} \mathbf{u}$ for the same rotational operation. These two modes are independent for a square wire, whose dispersion curves are denoted by the solid and dot-dashed lines in Fig. 1, respectively. The crossing of the dispersion curves is peculiar to the square wires. These modes are no longer independent but are coupled for rectangular wires, inducing separation of their dispersion curves. Figure 5 shows 


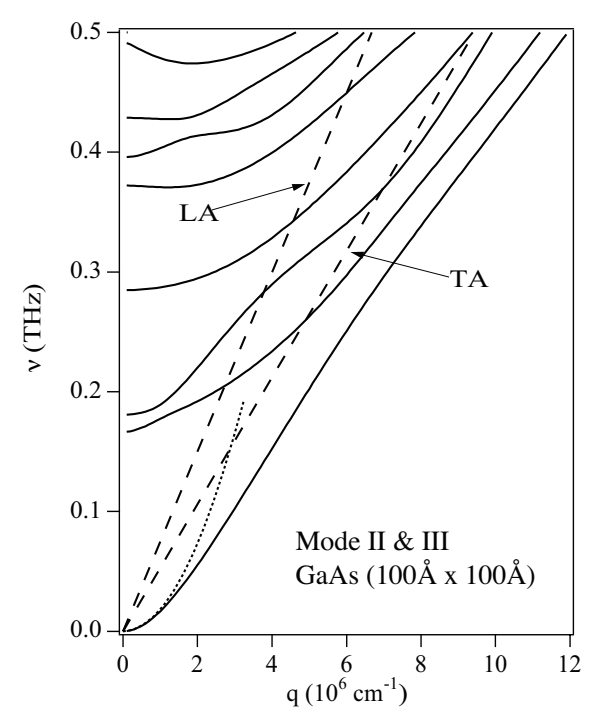

Figure 2. Dispersion curves of the flexural phonon modes of a GaAs square wire with cross sectional dimensions $100 \AA \times 100 \AA$. The dispersion relations of the two flexural modes are degenerate for a square wire. The solid lines denote the dispersion curves, and the dashed lines are the dispersion curves of the bulk LA and TA waves along the [001] direction. The dotted line denotes the dispersion curve of a bending wave in a rod, showing the parabolic dispersion relation in the long wavelength limit.

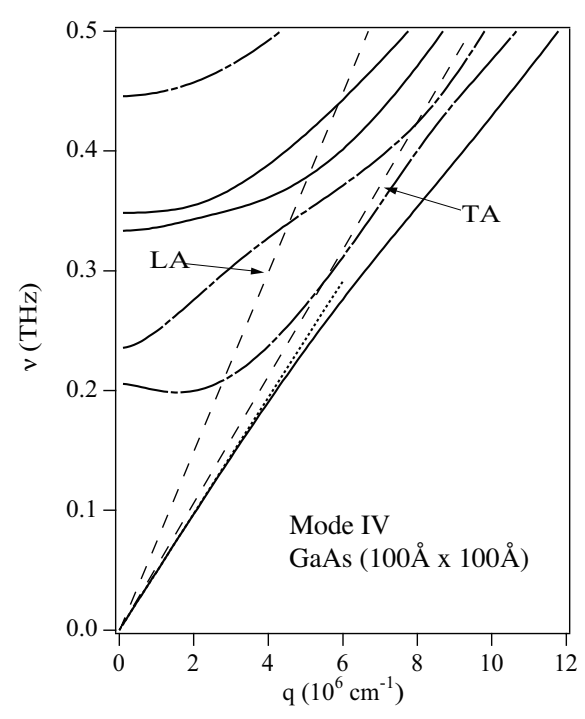

Figure 3. Dispersion curves of the torsional and shear phonon modes of a GaAs square wire with cross sectional dimensions $100 \AA \times 100 \AA$. The solid and dot-dashed lines denote the dispersion curves of the torsional and shear modes, respectively. The dashed lines are the dispersion curves of the bulk LA and TA waves along the [001] direction. The dotted line is the dispersion curve of a torsional wave in a rod in the long wavelength limit. 
(a)

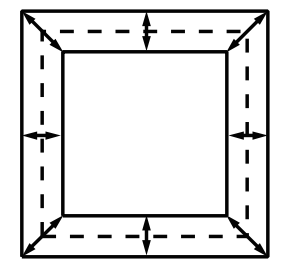

(b)

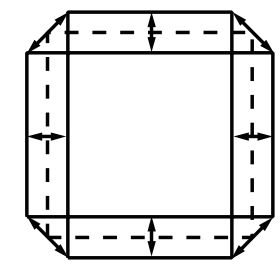

Figure 4. Schematic vibrational patterns of the dilatational mode: (a) the mode with the rotational symmetry $\mathbf{u}=C_{4} \mathbf{u}$ shows the simple dilatation and contraction of wire, while (b) the mode with $\mathbf{u}=-C_{4} \mathbf{u}$ exhibit the alternate dilatation and contraction of thickness of wire. The squares with dashed lines denote the undeformed cross section of wire, and the arrows denote the direction of displacement.

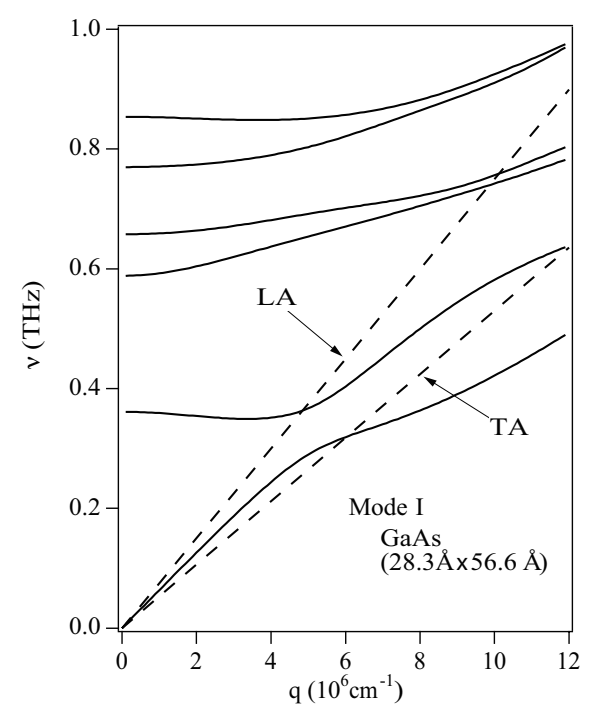

Figure 5. Dispersion curves of the dilatational phonon mode of a GaAs rectangular wire with cross sectional dimensions $28.3 \AA \times 56.6 \AA$. The solid lines denote the dispersion curves, and the dashed lines are the dispersion curves of bulk LA and TA waves along the [001] direction.

the dispersion curves of the dilatational mode of a wire with cross-sectional dimensions $28.3 \AA \times 56.6 \AA$, which was discussed in Ref. [10], showing that all the dispersion curves anticross at all frequencies studied.

Figure 6 shows the dispersion curves of the thickness and width modes of the wire. The lowest subband of the width mode is almost the same as that of the dilatational mode. The phonon modes of rectangular wires have been believed to be well described by the approximate hybrid modes referred to as the thickness and width modes[15, 16] assuming separable boundary conditions, in particular, for wires with the cross-sectional aspect ratio greater than two. We find poor agreement between the approximate hybrid modes and the dilatational mode for subbands other than the lowest. From these results, 


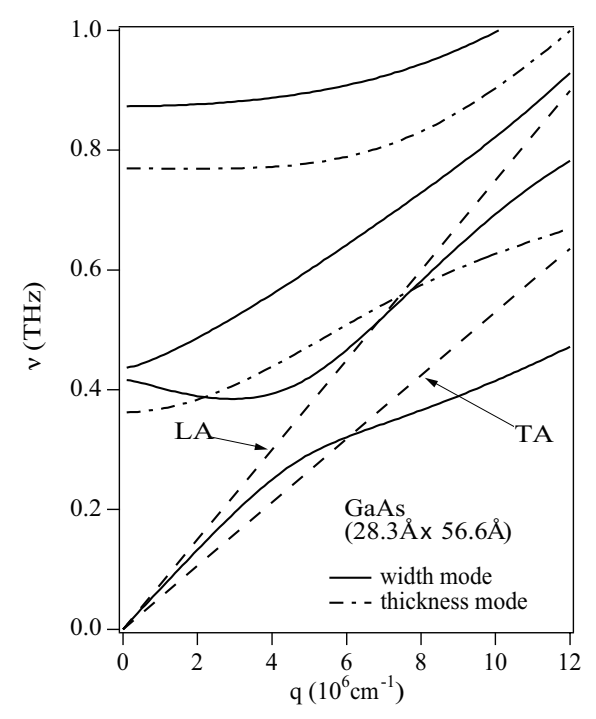

Figure 6. Dispersion curves of the thickness and width modes of a GaAs rectangular wire with cross sectional dimensions $28.3 \AA \times 56.6 \AA$. The solid and dot-dashed lines denote the dispersion curves of the width and thickness modes, respectively. The dashed lines are the dispersion curves of the bulk LA and TA waves along the [001] direction.

(a) (b)
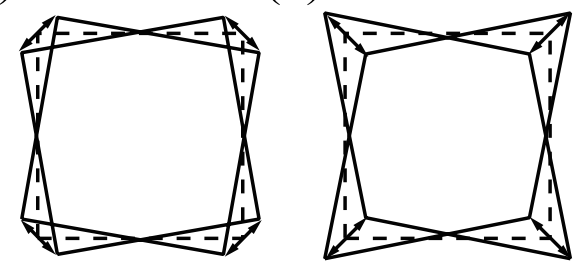

Figure 7. Schematic vibrational patterns of (a) the torsional mode with the rotational symmetry $\mathbf{u}=C_{4} \mathbf{u}$ and (b) the shear mode with $\mathbf{u}=-C_{4} \mathbf{u}$. The torsional mode shows rotation of wire about the wire axis, and the shear mode exhibits alternate stretching in the diagonal directions. The squares with dashed lines denote the undeformed cross section of wire, and the arrows denote the direction of displacement.

we conclude that the approximate hybrid modes are valid only for the lowest phonon subband.

Mode IV consists of the torsional and shear modes, as mentioned above. Vibrational patterns of these modes are schematically illustrated in Fig. 7(a) and 7(b), respectively. As understood from these figures, the displacement vector of the torsional mode is transformed as $\mathbf{u}=C_{4} \mathbf{u}$ and that of the shear mode is transformed as $\mathbf{u}=-C_{4} \mathbf{u}$ by the rotational operation about the wire axis. Their dispersion relations are denoted in Fig. 3 by the solid and dot-dashed lines, respectively. In contrast to the dilatational mode, although these modes are independent for a square wire, there is no intersection of the dispersion curves, at least below $0.5 \mathrm{THz}$. These two modes are not well defined for 
rectangular wires, but are mixed. However, the effects of the mode mixing on phonon subbands are expected to be small since their dispersions are separated and there is not degeneracy between them for a square wire. On the other hand, the change in wire geometry modulates the mechanical properties of wires such as the torsional rigidity and the moments of inertia, causing modifications in dispersion relations. As for the lowest subband, the dispersion curve is lowered with increasing aspect ratio and can be approximated, at a small $q$, by $\omega=\frac{2 H}{W} \sqrt{\frac{\mu}{\rho}} q$ for very flat rectangular wires $W>>H$, where $\mu$ is one of the Lamé constants. In addition, change in wire thickness affects the lateral wavevector quantization. Hence the dispersion relations of the higher subbands show complicated dependence on the cross sectional aspect ratio more than the lowest subbands.

As for the flexural modes, the dispersion relations of modes II and III are no longer degenerate for rectangular wires. The curvature of the lowest subbands of modes II and III at a small $q$ is proportional to $H$ and $W$, respectively. Interestingly, the dispersion relations of the lowest subband of mode II(III) depends, even for a large $q W(H)(<10)$, on only $H(W)$ as if the modes were Lamb waves in a free plate. In contrast, higher subbands are affected by the change in the lateral wavevector quantization as well as in mechanical properties of a wire, showing complex dependence on the wire dimensions more than the lowest subbands like mode IV.

\section{Edge modes}

Figures 1, 2, and 3 show that the lowest and following two dispersion curves of all the modes fall below the dispersion curve of the bulk TA waves along the [001] direction with increasing $q$, which leads to complex lateral wavevectors. As a result, acoustic waves will decay exponentially in the directions normal to the edges or surfaces of wires. Hence we may expect the existence of edge or surface modes for large $q$. To confirm that the vibrations are localized at the wire corners or surfaces, we investigated the spatial distribution of squared displacement vectors $|\mathbf{u}|^{2}$. Figure 8 (a) illustrates the $|\mathbf{u}|^{2}$ of the lowest subband of the dilatational mode at $q=8 \times 10^{6} \mathrm{~cm}^{-1}$ of the square wire with cross sectional dimensions $100 \AA \times 100 \AA$, showing conspicuously large amplitude at the wire corners. Large amplitude at the wire corners similar to Fig. 8(a) is also found for the second and third subbands of the dilatational mode, the lowest and second subbands of the flexural modes, and the lowest subbands of the torsional and shear modes. Figures $8(\mathrm{~b})$ and $8(\mathrm{c})$ illustrate the $|\mathbf{u}|^{2}$ of the lowest subbands of the flexural and torsional modes at $q=8 \times 10^{6} \mathrm{~cm}^{-1}$. We refer to the modes with large amplitude at the corners to as edge modes, although the amplitude does not completely vanish between the wire corners in contrast to the edge modes localized at edges or steps of infinite crystals.[18] The completely localized vibrations at the wire corners are found for huge wires or at an extremely large $q$.

In contrast to the edge modes, we have not obtained the apparent evidence of surface modes since the lowest subbands exhibit the characteristic decay of the edge 

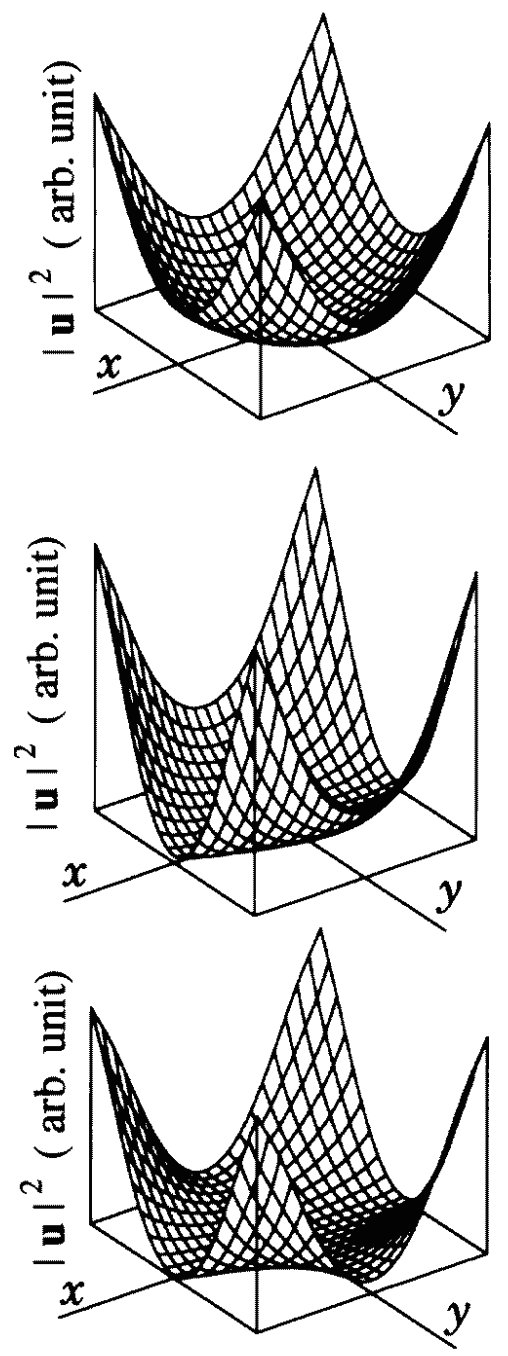

Figure 8. Spatial distribution of $|\mathbf{u}|^{2}$ of the lowest subband at $q=8 \times 10^{6} \mathrm{~cm}^{-1}$ of (a) the dilatational, (b) flexural, and (c) torsional modes of a GaAs square wire of $100 \AA \times 100 \AA$.

modes as mentioned above. However, surface and edge modes should coexist for huge wires. Then some of the edge modes are expected to change into surface modes with increasing wire dimensions.

\section{Phonon field}

The acoustic phonon field is described in terms of annihilation $a_{J}$ and creation operators $a_{J}^{\dagger}$ of phonons as

$$
\mathbf{u}_{o p}=\sum_{J}\left[a_{J} \mathbf{u}_{\mathbf{J}}+a_{J}^{\dagger} \mathbf{u}_{\mathbf{J}}^{*}\right],
$$


provided that the normal phonon modes $\mathbf{u}_{J}$ satisfy the orthonormal condition

$$
\int_{V} \rho \mathbf{u}_{J^{\prime}}^{*} \cdot \mathbf{u}_{J} d V=\frac{\hbar \delta_{J, J^{\prime}}}{2 \omega_{J}}
$$

as well as the completeness condition

$$
\rho \sum_{J} \omega_{J} u_{J, i}(\mathbf{r}) u_{J, j}\left(\mathbf{r}^{\prime}\right)=\frac{\hbar}{2} \delta_{i, j} \delta\left(\mathbf{r}-\mathbf{r}^{\prime}\right) .
$$

Here $J$ denotes the phonon modes discussed above, frequencies and the longitudinal wavevectors.

The derived normal modes from Eq. (11) satisfy the orthogonal condition. Considering that the $\boldsymbol{\Gamma}$ is a hermitian matrix and $\chi_{J}$ is an eigenvector of Eq. (11) for an eigenvalue $\omega_{J}^{2}$, we obtain for two normal modes $J$ and $J^{\prime}$,

$$
\left(\omega_{J}^{2}-\omega_{J^{\prime}}^{2}\right) \chi_{J^{\prime}}^{\dagger} \mathbf{E} \chi_{J}=0 \text {. }
$$

For the non-degenerate case, $\omega_{J} \neq \omega_{J^{\prime}}$, it is apparent that

$$
\chi_{J^{\prime}}^{\dagger} \mathbf{E} \chi_{J}=0 \text {. }
$$

Substituting Eq. (6) into (16) and integrating over the volume, the left hand side of Eq. (16) equals $V \boldsymbol{\chi}_{J^{\prime}}^{\dagger} \mathbf{E} \boldsymbol{\chi}$. Hence the normal modes are orthogonal for the nondegenerate case. For the degenerate case, $\omega_{J}=\omega_{J^{\prime}}$, we can show the orthogonality, considering the spatial symmetries of the normal modes. As an example, let us consider the case that the dispersion curves of the dilatational modes intersect. As discussed above, the crossing of the dispersion curves occurs for a square wire, and the normal modes have the distinct rotational symmetries along the wire axis, i.e. $\mathbf{u}_{1}=C_{4} \mathbf{u}_{1}$ and $\mathbf{u}_{2}=-C_{4} \mathbf{u}_{2}$. For the square wire, the following equality for an arbitrary function $f(\mathbf{r})$ holds

$$
\int_{V} f(\mathbf{r}) d \mathbf{r}=\int_{V}\left[C_{4} f(\mathbf{r})\right] d \mathbf{r} .
$$

Because the product $\mathbf{u}_{1} \cdot \mathbf{u}_{2}$ changes its sign for the rotational operation $C_{4}$, the integration of the product $\mathbf{u}_{1} \cdot \mathbf{u}_{2}$ vanishes. Using the rotational symmetries and the parity of the normal modes, we can also prove the orthogonality among the four kinds of normal modes when their dispersion curves intersect, as well as for rectangular wires. Thus the orthogonal condition (16) is proved to be satisfied.

Putting $J=J^{\prime}$, we obtain the normalization condition for the coefficients $\chi_{J, i \lambda}$ from Eqs. (6) and (16)

$$
\chi_{J}^{\dagger} \mathbf{E} \chi_{J}=\frac{1}{W H L_{w}} \frac{\hbar}{2 \omega_{J}}
$$

where $L_{w}$ is the wire length. The coefficients $\chi_{J, i \lambda}$ should satisfy the normalization condition (21) as well as the eigenvalue equation (11) for the normalized phonon modes. In practice the coefficients $\chi_{J, i \lambda}$ numerically obtained from Eq. (11) are scaled up or down so that they satisfy the normalization condition (21). Introducing a factor $\alpha(>0)$, 
we define the displacement components $u_{J, i}$ satisfying the normalization condition (16) by

$$
u_{J, i}=\alpha \chi_{J, i \lambda} \Phi_{\lambda}
$$

instead of (6). The factor $\alpha$ can be readily obtained by substituting Eq. (22) into (16), and the normalized displacement components $u_{J, i}$ are expressed by

$$
u_{J, i}=\frac{1}{\sqrt{W H L_{w}}} \sqrt{\frac{\hbar}{2 \omega_{J}}} \frac{\chi_{J, i \lambda}}{\sqrt{\chi_{J}^{\dagger} \mathbf{E} \chi_{J}}} \Phi_{\lambda}
$$

in terms of the eigenvectors $\chi_{J}$ of Eq. (11) corresponding to the normal modes.

In contrast to the orthogonal condition, it is difficult to prove the completeness of the normal modes in terms of the solutions of Eq. (11). However, completeness should be satisfied if all the normal modes are included in the summation of Eq. (17). In this work, we have investigated all possible phonon modes with spatial symmetries related to a rectangular wire. We have confirmed the expected bulk modes and have identified a previously overlooked edge mode. Therefore we anticipate that the normal modes obtained in this paper satisfy the completeness (17).

Thus the phonon field can be expressed in terms of the normalized phonon modes (23) and their complex conjugate. The Hamiltonian of phonons is diagonalized using Eqs. (15) and (23). These two equations can also be used to derive the deformation and piezoelectric potential Hamiltonians, and the ripple mechanism[19, 20] as will be discussed elsewhere.

\section{Summary}

The acoustic phonon modes of Q1D rectangular wires of cubic crystals have been precisely derived by using the $x y z$ algorithm used in the resonant ultrasound spectroscopy. The acoustic phonon modes are classified according to their spatial symmetries into a compressional mode termed the dilatational mode and noncompressional modes referred to as the torsional, shear, and two flexural modes. The dispersion relations of the normal modes were obtained for GaAs square and rectangular wires, exhibiting the subband structures. The lowest subbands of these modes reduce to the vibrational modes of a rod in the long wavelength limit, showing the linear or parabolic dispersion relations with respect to a longitudinal wavevector $q$ peculiar to each mode. There are intersections among the dispersion relations of the dilatational mode because the dilatational mode is divided into two independent modes for square wires. For a rectangular wire, mode coupling between the dilatational modes and between the torsional and shear modes take place. The mode coupling substantially modifies the dispersion relations of the dilatational mode, separating their dispersion curves, while the coupling between the torsional and shear modes does not have significance for phonon spectra in comparison with the change in mechanical properties. We also examined the validity of the approximate hybrid modes by comparing the dispersion 
relations for a rectangular wire, and found that only the lowest subband can be well described by the width mode. We confirm that the lowest subbands are the acoustic edge modes which decay in the directions normal to the wire corners. Finally, applying the normalization condition to the phonon modes, we formulated the phonon field.

In this paper we have demonstrated that the acoustic phonon modes of square and rectangular cross section, Q1D wires can be precisely derived. The method used is general and can be applied to free-standing structures with other cross sectional profiles, for instance triangular. [6] Experimental evidence for reduced dimensionality phonons has been reported in supported metal wires. [7, 8] The coupling of such wires to a substrate will cause the phonon spectrum to be different from that of the free-standing wires reported here. We anticipate that with some modification the $x y z$ algorithm will be applicable to supported structures, which will enable a comparison between the predicted and observed phonon spectra. Work towards this application is underway and will be reported elsewhere.

The authors would like to thank Professor H. J. Maris for valuable advice on this work. This work was partly supported by a Grant-in-Aid for Scientific Research(C) from the Ministry of Education, Science, and Culture, Japan (Grant No. 08650061). MNW was supported in part by the National Science Foundation under Grant No. DMR-9501108. 


\section{Appendix A. Matrix $\Gamma$}

The matrix elements $\Gamma_{i \lambda^{\prime} ; j \lambda}$ are:

$$
\begin{aligned}
\Gamma_{1 \lambda^{\prime} ; 1 \lambda}= & \frac{4}{W^{2}} C_{11} m m^{\prime} F\left(m+m^{\prime}-2 ; n+n^{\prime}\right)+\frac{4}{H^{2}} C_{44} n n^{\prime} F\left(m+m^{\prime} ; n+n^{\prime}-2\right) \\
& +C_{44} q^{2} F\left(m+m^{\prime} ; n+n^{\prime}\right) \\
\Gamma_{1 \lambda^{\prime} ; 2 \lambda}= & \frac{4}{W H}\left(C_{12} m^{\prime} n+C_{44} m n^{\prime}\right) F\left(m+m^{\prime}-1 ; n+n^{\prime}-1\right) \\
\Gamma_{1 \lambda^{\prime} ; 3 \lambda}= & -\frac{2 i}{W} q\left(C_{44} m-C_{12} m^{\prime}\right) F\left(m+m^{\prime}-1 ; n+n^{\prime}\right) \\
\Gamma_{2 \lambda^{\prime} ; 1 \lambda}= & \frac{4}{W H}\left(C_{44} m^{\prime} n+C_{12} m n^{\prime}\right) F\left(m+m^{\prime}-1 ; n+n^{\prime}-1\right) \\
\Gamma_{2 \lambda^{\prime} ; 2 \lambda}= & \frac{4}{W^{2}} C_{44} m m^{\prime} F\left(m+m^{\prime}-2 ; n+n^{\prime}\right)+\frac{4}{H^{2}} C_{11} n n^{\prime} F\left(m+m^{\prime} ; n+n^{\prime}-2\right) \\
& +C_{44} q^{2} F\left(m+m^{\prime} ; n+n^{\prime}\right) \\
\Gamma_{2 \lambda^{\prime} ; 3 \lambda}= & -\frac{2 i}{H} q\left(C_{44} n-C_{12} n^{\prime}\right) F\left(m+m^{\prime} ; n+n^{\prime}-1\right) \\
\Gamma_{3 \lambda^{\prime} ; 1 \lambda}= & -\frac{2 i}{W} q\left(C_{12} m-C_{44} m^{\prime}\right) F\left(m+m^{\prime}-1 ; n+n^{\prime}\right) \\
\Gamma_{3 \lambda^{\prime} ; 2 \lambda}= & -\frac{2 i}{H} q\left(C_{12} n-C_{44} n^{\prime}\right) F\left(m+m^{\prime} ; n+n^{\prime}-1\right) \\
\Gamma_{3 \lambda^{\prime} ; 3 \lambda=} & \frac{4}{W^{2}} C_{44} m m^{\prime} F\left(m+m^{\prime}-2 ; n+n^{\prime}\right)+\frac{4}{H^{2}} C_{44} n n^{\prime} F\left(m+m^{\prime} ; n+n^{\prime}-2\right) \\
& +C_{11} q^{2} F\left(m+m^{\prime} ; n+n^{\prime}\right),
\end{aligned}
$$

\section{Appendix B. Displacement components}

The displacement components are:

$$
\begin{aligned}
& u_{x}=\sum_{r, s=0}^{2(r+s)+1 \leq N} \chi_{1 r s}\left(\frac{2 x}{W}\right)^{2 r+1}\left(\frac{2 y}{H}\right)^{2 s} e^{i q z} \\
& u_{y}=\sum_{r, s=0}^{2(r+s)+1 \leq N} \chi_{2 r s}\left(\frac{2 x}{W}\right)^{2 r}\left(\frac{2 y}{H}\right)^{2 s+1} e^{i q z} \\
& u_{z}=\sum_{r, s=0}^{2(r+s) \leq N} \chi_{3 r s}\left(\frac{2 x}{W}\right)^{2 r}\left(\frac{2 y}{H}\right)^{2 s} e^{i q z}
\end{aligned}
$$

for mode I,

$$
\begin{aligned}
& u_{x}=\sum_{r, s=0}^{2(r+s)+2 \leq N} \chi_{1 r s}\left(\frac{2 x}{W}\right)^{2 r+1}\left(\frac{2 y}{H}\right)^{2 s+1} e^{i q z} \\
& u_{y}=\sum_{r, s=0}^{2(r+s) \leq N} \chi_{2 r s}\left(\frac{2 x}{W}\right)^{2 r}\left(\frac{2 y}{H}\right)^{2 s} e^{i q z}
\end{aligned}
$$




$$
u_{z}=\sum_{r, s=0}^{2(r+s)+1 \leq N} \chi_{3 r s}\left(\frac{2 x}{W}\right)^{2 r}\left(\frac{2 y}{H}\right)^{2 s+1} e^{i q z}
$$

for mode II,

$$
\begin{aligned}
& u_{x}=\sum_{r, s=0}^{2(r+s) \leq N} \chi_{1 r s}\left(\frac{2 x}{W}\right)^{2 r}\left(\frac{2 y}{H}\right)^{2 s} e^{i q z} \\
& u_{y}=\sum_{r, s=0}^{2(r+s)+2 \leq N} \chi_{2 r s}\left(\frac{2 x}{W}\right)^{2 r+1}\left(\frac{2 y}{H}\right)^{2 s+1} e^{i q z} \\
& u_{z}=\sum_{r, s=0}^{2(r+s)+1 \leq N} \chi_{3 r s}\left(\frac{2 x}{W}\right)^{2 r+1}\left(\frac{2 y}{H}\right)^{2 s} e^{i q z}
\end{aligned}
$$

for mode III, and

$$
\begin{aligned}
& u_{x}=\sum_{r, s=0}^{2(r+s)+1 \leq N} \chi_{1 r s}\left(\frac{2 x}{W}\right)^{2 r}\left(\frac{2 y}{H}\right)^{2 s+1} e^{i q z} \\
& u_{y}=\sum_{r, s=0}^{2(r+s)+1 \leq N} \chi_{2 r s}\left(\frac{2 x}{W}\right)^{2 r+1}\left(\frac{2 y}{H}\right)^{2 s} e^{i q z} \\
& u_{z}=\sum_{r, s=0}^{2(r+s)+2 \leq N} \chi_{3 r s}\left(\frac{2 x}{W}\right)^{2 r+1}\left(\frac{2 y}{H}\right)^{2 s+1} e^{i q z}
\end{aligned}
$$

for mode IV.

\section{References}

[1] Auld B A 1973Acoustic Fields and Waves in Solids (Wiley, New York) Vol. II Chap. 10

[2] Kelly M J 1982 J. Phys. C.: Solid State Phys. 15 L969

[3] Ishii K 1973 Prog. Theor. Phys. Suppl. 5377

[4] Smith C G and Wybourne M N 1986 Solid State Commun. 57411

[5] Jäckle J 1981 Solid State Commun. 391261

[6] Potts A, Kelly M J, Smith C G, Hasko D G, Cleaver J R A, Ahmed H, Peacock D C, Ritchie D A, Frost J E F and Jones G A C 1990 J. Phys.: Condens Matt. 21817

[7] Seyler J and Wybourne M N 1992 Phys. Rev. Lett. 691427

[8] Wybourne M N 1996 Physica B219 \& 22013

[9] Nishiguchi N 1995 Phys. Rev. B52 5279

[10] Segi Y, Kim K W, Stroscio M A, Iafrate G J and Ballato A 1994 Phys. Rev. B50 1733

[11] Bannov N A, Mitin V V, and Stroscio M A 1995 Quantum Transport in Ultrasmall Devices, editors Ferry D K, Grubin H L, Jacoboni C, and Jauho A-P, (Plenum, New York) p191

[12] Demarest H H Jr. 1971 J. Acoust. Soc. Am. 49768

[13] Visscher W M, Migliori A, Bell T M and Reinert R A 1991 J. Acoust. Soc. Am. 902154

[14] Maynard J 1996 Physics Today Jan. 26 and references quoted therein

[15] Morse R W 1948 J. Acoust. Soc. Am. 20833

[16] Morse R W 1950 J. Acoust. Soc. Am. 22219

[17] Landau L D, Lifshitz E M, Kosevich A M and Pitaevskii L P 1986 Theory of Elasticity (Pergamon, New York) 3rd ed. Chap. 12

[18] Maradudin A A, Wallis R F, Mills D L and Ballard R L 1972 Phys. Rev. B6 1106 
[19] Vasko F. T. and Mitin V V 1995 Phys. Rev. B52 1500

[20] Knipp P. A. and Reinecke T L 1995 Phys. Rev. B52 5923

[21] Adachi S 1985 J. Appl. Phys. 58 R1 